\title{
Large-Scale Screening of Lipase Acid Deficiency in at Risk Population
}

Abdellah Tebani ${ }^{1}$, Bénédicte Sudrié-Arnaud ${ }^{1}$, Hela Boudabous ${ }^{2}$, Anais Brassier ${ }^{3}$, Rodolphe Anty ${ }^{4}$, Sarah Snanoudj 1, Armand Abergel 5, Marie-Thérèse Abi Warde 6, Edouard Bardou-Jacquet ${ }^{7}$, Reda Belbouab ${ }^{8}$, Eloi Blanchet 9 , Corinne Borderon ${ }^{10}$, Jean-Pierre Bronowicki ${ }^{11}$, Bertrand Cariou ${ }^{12}$, Claire Carette ${ }^{13}$, Myriam Dabbas ${ }^{14}$, Hélène Dranguet ${ }^{1}$, Victor de Ledinghen ${ }^{15}$, Jean Ferrières ${ }^{16}$, Maeva Guillaume ${ }^{17}$, Michel Krempf ${ }^{18}$, Florence Lacaille ${ }^{19}$, Dominique Larrey ${ }^{20}$, Vincent Leroy ${ }^{21}$, Marietta Musikas ${ }^{22}$, Eric Nguyen-Khac ${ }^{23}$, Denis Ouzan ${ }^{24}$, Jean-Marc Perarnau 25, Carine Pilon 1, Vlad Ratzlu 26, Alice Thebaut ${ }^{27}$, Thierry Thevenot ${ }^{28}$, Isabelle Tragin 1, Valérie Triolo ${ }^{29}$, Bruno Vergès ${ }^{30}$, Sabrina Vergnaud ${ }^{31}$, Soumeya Bekri ${ }^{1 *}$

1 Normandie Univ, UNIROUEN, INSERM U1245, CHU Rouen, Department of Metabolic Biochemistry, 76000 Rouen, France

2 Pediatric Department, La Rabta Hospital, Faculty of Medecine of Tunis, University of Tunis El Manar, Jabberi, Jebal Lakhdhar, Tunis, Tunisia

3 Reference Center of Inherited Metabolic Diseases, Necker Enfants Malades Hospital, Imagine Institute, University Paris Descartes, AP-HP, 75015 Paris, France

4 INSERM, U1065, C3M, Team 8 "Hepatic Complications in Obesity", Nice, France; Université Côte d'Azur, Nice, France; CHU of Nice, Digestive Center, Nice, France

5 Department of Digestive Medicine, CHU Estaing, Clermont-Ferrand, France; Unité mixte de recherche Université d'Auvergne CNRS 6284, France

6 Pediatric Neurology Department, CHU Strasbourg, France; marie-therese.abi-warde@chru-strasbourg.fr (M.T.A.)

7 Univ Rennes, INSERM, Institut Numecan, Liver disease unit, CHU de Rennes, F-35000 Rennes, France;

8 Pediatric Department, University Hospital Center Mustapha Bacha, 16000 Algiers, Algeria

9 Service Hépatologie-Gastroenterologie, Groupe Hospitalier La Rochelle-Ré-Aunis, La Rochelle, France

10 Clermont-Ferrand University Hospital, Clermont-Ferrand, France

11 Department of Hepato-Gastroenterology, Centre Hospitalo-Universitaire de Nancy, 54000 Nancy, France

12 Université de Nantes, CHU de Nantes, CNRS, INSERM, L'institut du thorax, Department of Endocrinology-Diabetology-Nutrition, F-44000 Nantes, France

13 AP-HP, Department of Nutrition, Centre spécialisé de l'Obesité Hôpital Européen Georges Pompidou, Paris University, Paris, France

14 AP-HP, Nutrition Obesity Unit, Necker Hospital, Paris, France

15 Hepatology Unit, University Hospital, CHU Bordeaux, Pessac, France

16 Department of Cardiology and UMR INSERM 1027, Toulouse University School of Medicine, Toulouse, TSA 50032 31059, France

17 Service d'Hépatologie CHU Toulouse Rangueil, Institut Cardiomet et Université Paul Sabatier, Toulouse, France

18 Endocrinology, Metabolic diseases and Nutrition, ELSAN, Clinique Breteché, Nantes, France

19 Gastroenterology Hepatology Nutrition Unit, Hôpital Necker-Enfants Malades, Paris, France

${ }^{20}$ Liver and Transplantation Unit, Montpellier School of Medicine and IRB-INSERM-1183, Montpellier, France

${ }^{21}$ Service d'Hépato-Gastroentérologie, Centre Hospitalier Universitaire Grenoble-Alpes, INSERM U1209, Université Grenoble-Alpes, Grenoble, France

22 Department of Hepato-Gastroenterology and Nutrition, Caen University Hospital, France

${ }^{23}$ Service d'Hépato-Gastroentérologie, Amiens University Hospital, and Equipe Région INSERM 24, University of Picardy, Amiens, France

24 Institut Arnaud Tzanck, Service d'Hépatologie, St Laurent du Var, France

25 Service d'Hépato-gastroentérologie, Centre Hospitalo-Universitaire, Tours, France

26 Department of Hepatology, Groupe Hospitalier Pitié-Salpêtrière, Assistance Publique Hopitaux de Paris, Paris, France; University Pierre et Marie Curie, Institut National de la Santé et de la Recherche Médicale UMR 938, Paris, France

27 Pediatric Hepatology \& Pediatric Liver Transplant Department, Centre de Référence de l'Atrésie des Voies Biliaires et des Cholestases Génétiques (AVB-CG), Filière de Santé des Maladies Rares du Foie de l'enfant et de l'adulte (FILFOIE), European Reference Network RARE-LIVER, Assistance Publique-Hôpitaux de Paris, Faculty of Medecine Paris-Saclay, CHU Bicêtre, Le Kremlin-Bicêtre, France

28 Centre Hospitalier Universitaire de Besançon, Hôpital Jean Minjoz, Service d'Hépatologie et de Soins Intensifs Digestifs, Besançon, France

${ }^{29}$ CHU de Nice, Hôpital Lenval, Nice, France

30 Université de Bourgogne, Centre de Recherche INSERM LNC-UMR1231; Service de Diabétologie et Endocrinologie, CHU François Mitterand, BP 77908, Dijon cedex 21079, France

31 Department of Biochemistry Toxicology and Pharmacology, Grenoble University Hospital, La Tronche, France

* Correspondence:

Prof. Soumeya Bekri

Department of Metabolic Biochemistry

Rouen University Hospital

37 Boulevard Gambetta, 76000 Rouen, France.

Phone: + (33) 232888124

soumeya.bekri@chu-rouen.fr 


\section{Abstract}

Background

Lysosomal acid lipase deficiency (LALD, OMIM\#278000) is a rare lysosomal disorder with an autosomal recessive inheritance. The main clinical manifestations are related to a progressive accumulation of cholesteryl esters, triglycerides or both within the lysosome in different organs such as the liver, spleen, and cardiovascular system. A wide range of clinical severity is associated with LALD including a severe very rare antenatal/neonatal/infantile phenotype named Wolman disease and a late-onset form named cholesteryl ester storage disease (CESD).

Methods

This study aimed to investigate a cohort of at-risk patients (4174) presenting with clinical or biological signs consistent with LALD using the assessment of LAL activity on dried blood spots.

Results

LAL activity was lower than $0.05 \mathrm{nmol} /$ punch/L (cut-off: 0.12 ) in 19 patients including 13 CESD and 6 Wolman. Molecular study has been conducted in 17 patients and succeeded in identifying 34 mutated alleles. Fourteen unique variants have been characterized, 7 of which are novel.

Conclusion

This study allowed to identify a series of patients and expanded the molecular spectrum knowledge of LALD. Besides, a new screening criteria grid based on the clinical/biological data from our study and the literature has been proposed in order to enhance the diagnosis rate in at risk populations.

Keywords: LAL; Acid lipase deficiency; screening; Wolman; CESD; Cholesterol ester storage disease; Dried blood spot; DBS 


\section{Introduction}

Lysosomal acid lipase (LAL - EC.3.1.13) acts mainly in the hepatocytes following low-density lipoproteins (LDL) endocytosis to hydrolyze cholesterol esters (CE) and triglycerides (TG) into glycerol, free cholesterol (FC) and fatty acids. The reaction products are released into the cytosol and are involved in different metabolism such as energy, transport and anabolism [1]. FC is involved in several regulatory pathways. Sterol regulatory element binding proteins (SREBPs) modulate the expression of several genes involved in cholesterol and lipid synthesis. After their translation in the endoplasmic reticulum (ER), SREBPs form a complex with SREBP Cleavage Activating Proteins (SCAPs). In the presence of high FC concentrations, SCAP maintains SREBP in the ER. Conversely, in the presence of low cytosolic FC concentrations, SCAP brings SREBP to the Golgi apparatus where it becomes active through proteolytic cleavage steps. Then, SREBP translocates into the nucleus and binds to specific sequences located in the 5'UTR/enhancer regions on the target genes named SRE for Sterol Response Elements [2]. ABCA1, is an ATPBinding-Cassette transporter that mediates the transport of cytosolic FC and phospholipids to pre-High-Density Lipoprotein (pre-HDL) particles to form HDLs [3].

LAL deficiency (LALD, OMIM\#278000) is a rare autosomal recessive lysosomal disorder. The decrease or the loss of enzyme activity leads to a progressive accumulation of the enzyme substrates namely, CE, TG or both within the lysosome in different organs mainly the liver, spleen, and cardiovascular system $[1,4]$. In LALD, intracellular FC depletion induces through SREBP regulation (i) an increase of cholesterol and apolipoprotein B synthesis with a subsequent increase of Very Low-Density Lipoproteins (VLDL) secretion, (ii) an increase of LDL receptor expression with a subsequent increase of LDL uptake. Besides, the low cytosolic FC concentration prevents ABCA1-mediated HDL particle formation. As a result, the lipid profile in LALD displays a high total cholesterol, LDL and TG concentrations, and a decreased HDL level [1, 4].

This lipid accumulation causes several cellular damages. In the liver, microvacuolar steatosis, elevation of transaminase activity, fibrosis and cirrhosis have been reported. Splenomegaly, the subsequent anemia and thrombocytopenia are probably related to lipid build-up within the macrophages. Lipid accumulation in the intestinal mucosa induces several gastrointestinal 
disturbances such as diarrhea, abdominal and epigastric pain. Steroid hormones are synthesized in the adrenal cortex from FC. In LALD, low FC hampers the steroid synthesis and results in adrenal insufficiency. In severe antenatal forms of LALD, adrenal calcification related to extensive fetal adrenal necrosis can be detected by ultrasound [5]. The dyslipidemia associated to LALD underlies the increased cardiovascular risk.

LALD, as all inborn errors of metabolism (IEMs), is primarily due to a monogenic alteration. But it is to be noted that a large number of genetic and environmental factors may modulate phenotypic expression. Thus, a wide range of clinical severity is associated with LALD. This continuum encompasses a severe antenatal/neonatal/infantile phenotype named Wolman disease and a late-onset form named cholesteryl ester storage disease (CESD) [1].

Wolman disease is an ultra-rare condition (incidence: $1 / 500,000$ ) with a very severe outcome. Prior to enzymotherapy treatment survival expectancy was less than 2 years [6]. The symptom onset may occur in the fetal stage with $50 \%$ of affected infants presenting with antenatal adrenal calcifications. Massive hepatomegaly and failure to thrive with malabsorption contribute to the premature death.

CESD incidence is estimated at 1/40,000 [7]. Hepatomegaly is often present at diagnosis and lysosomal lipid accumulation leads to progressive organ damages including cardiovascular alteration, fibrosis and cirrhosis. Recently, the prevalence at birth of LAL deficiency has been estimated at 3.45-5.97 cases per million births in European-ancestry populations [8]

At the molecular level, LALD results from the LIPA gene alterations. This gene is located on chromosome 10q23.2 and includes 10 exons. To date, 111 LIPA variants have been reported $[1,4]$ with a splicing variant being frequently present (NM_000235:c.894G>A - p.(Gln298=)) [9].

LALD treatment used to be based on low-fat diet, lipid reduction and hematopoietic stem cell transplant or liver transplantation with variable outcome. An enzyme replacement therapy (ERT), Sebelipase alfa, has been recently approved considering the clinical benefit especially in the most severe cases with a significant survival increase [6]. 
This study reports the screening of a cohort of at-risk patients presenting with clinical or biological signs consistent with LALD.

\section{Materials and Methods}

\subsection{Cohort description}

This cohort is based on 4174 dried blood spot (DBS) samples obtained from individuals, who were suspected of having LALD after clinical screening (the presence of unexplained hepatomegaly or an increase in transaminase activity by $\geq 1.5$ times the upper reference limit, or a disturbed serum lipid profile with or without splenomegaly or gastrointestinal dysfunction or hepatic microvacuolar steatosis/fibrosis/cirrhosis), and submitted to our laboratory for the assessment of LAL activity between 2015 and 2019. The cohort included 1690 females $($ age $=42.78$ \pm 22.56 years) and 2494 males (age $=44.88 \pm 20.49$ years). Informed consents were obtained from all subjects involved in the study. Seventeen written informed consents have been signed for the molecular analysis.

\subsection{Lysosomal acid lipase}

All described laboratory procedures were performed at the Metabolic Biochemistry Department, Rouen University Hospital, Rouen, France

For all analyses presented in this study, DBS were prepared by spotting EDTA venous blood on filter paper (PerkinElmer 226). Fresh DBS were analyzed within three days upon preparation and were archived at $-20 \mathrm{C}$ in individual plastic bags with a desiccant.

The enzymatic assay was carried out based on the method described by Hamilton et al [10, 11] using 4-methylumbelliferyl palmitate (4-MU) as a substrate and the LAL inhibitor, Lalistat-2. Briefly, DBS was incubated in $200 \mu \mathrm{L}$ of water for 1 hour. Then, $40 \mu \mathrm{L}$ aliquots of DBS water extracts were preincubated with $10 \mu \mathrm{L}$ of $30 \mu \mathrm{M}$ Lalistat-2 or water for 10 minutes in a 96-well flat bottom black plate. Then, $150 \mu \mathrm{L}$ of reaction buffer solution containing 4-MU-Palm $(0.35 \mathrm{mM})$ and cardiolipin $(0.032 \%)$ in a $0.15 \mathrm{M}$ acetate buffer with $1 \%$ Triton X-100, pH 4.0 was added. The plate was sealed and incubated at $37{ }^{\circ} \mathrm{C}$ for 3 hours. Following the incubation, ten 4-MU standards serially diluted in water (Range: 0.02 - $11.34 \mu \mathrm{mol} 4-\mathrm{MU}$ ) were added to the plate. 
Then, $100 \mu \mathrm{L}$ of $0.15 \mathrm{M}$ acetate buffer with $1 \%$ Triton X-100 was added to all wells, and the fluorescence was measured in a plate reader (F7000, Hitachi, Japan) (excitation $\lambda=320 \mathrm{~nm}$; emission $\lambda=446 \mathrm{~nm}$ ). LAL activity was calculated by subtracting the value in the inhibited reaction (with Lalistat-2) from the value of reaction without the inhibitor (total lipase activity). The reference range was between 0.32 and $2.08 \mathrm{nmol} /$ punch/1 hour with a cut-off set at 0.12 for LALD.

\subsection{Molecular Analysis}

DNA extraction: for NGS analysis, genomic DNAs were extracted from blood using a silicamembrane-based DNA purification method (QIAamp DNA Blood Mini Kit, QIAGEN). NGS sequencing was performed at the IRIB-Rouen University Hospital Facility (Service Commun de Génomique).

The promoter region and the flanking intronic sequences of the LIPA gene (NM_000235) are included in our in-house lysosomal disease NGS panel. The NGS sequencing method has been reported previously [12]. Briefly, custom primers were designed using the SureDesign software (Agilent Technologies, Santa Clara, California, USA). The library preparation protocol was set up using the QXT SureSelect enrichment kit from Agilent. Library construction was done using enzymatic fragmentation and the SureSelectQXT kit (Agilent Technologies, Santa Clara, California, USA) to capture targeted sequences. Patients' libraries were pooled after the enrichment step. Libraries were sequenced on a MiSeq or a NextSeq 500 platform (Illumina, San Diego, USA) using 2x150 bp paired-end sequencing. For the detection of SNVs, indels and CNV, a double bioinformatics pipeline was used with complementary algorithms in order to optimize the disease-causing variant detection rate.

An in-house software, CanDiD allowed for the prioritization and filtration of variants using defined criteria such as minor allele frequency in public databases or consequences of the variant (missense, synonym, nonsense, splicing) [13]. The filtered variants were compared to variant databases including dbSNP (https://www.ncbi.nlm.nih.gov/snp/), GnomAD (https://gnomad.broadinstitute.org/), HGMD (http://www.hgmd.cf.ac.uk/) and LOVD (https://databases.lovd.nl/shared/genes). 
Evaluation of the pathogenicity of the variants were analyzed with in silico tools such as SIFT [14], PolyPhen2 [15] or MutationTaster [16] and M-CAP [17] to predict potential deleterious effect on protein function, and HumanSplicingFinder 2.4.1 [18], MaxEntScan [19], NNSPLICE [20], GeneSplicer [21], SpliceSiteFinder [22], and ESEFinder [23] for possible effect on splicing. Variant classification was done according to the recommendations of the American College of Medical Genetics [24].

The control of the sample identity was performed using a multiplex SNaPshot analysis comparing five SNPs located within the captured regions of 5 genes unrelated to LDs included in the panel.

\section{Results}

\subsection{Patients}

We assessed 4174 DBS samples (Supplementary Table S1). In females ( $n=1,690,40 \%$ of the cohort), the overall mean age was $42.78 \pm 22.56$ years, with a mean LAL activity of $0.98 \pm 0.59$ nmol/punch/1 hour. For males ( $\mathrm{n}=2,494,60 \%$ of the cohort), mean age was $44.88 \pm 20.49$ years, with a mean LAL activity of $0.89 \pm 0.54 \mathrm{nmol} / \mathrm{punch} / 1$ hour. One hundred and fifty-seven samples came from children below 2 years old (3.7\%) including 73 females and 84 males. Four thousand and seventeen (96.3\%) samples came from patients older than 2 years old and included 1607 females and 2410 males.

Based on this cohort, 19 patients (Figure 1 and 2) have been detected with an LAL activity lower than $0.05 \mathrm{nmol} / \mathrm{punch} / 1$ including 13 CESD (9 females and 4 males) with an average age at diagnosis of $22.42 \pm 18.23$ years and 6 Wolman ( 4 females and 2 males) with an average age at diagnosis of $3.12 \pm 4.08$ months. For CESD patients, laboratory work-up showed an impaired lipid profile (Supplementary Table S2) with a total cholesterol $=2.72 \pm 0.47 \mathrm{~g} / \mathrm{L}(<2.3)$, HDL cholesterol $=0.35 \pm 0.09 \mathrm{~g} / \mathrm{L}(\mathrm{N}>0.45), \mathrm{LDL}$ cholesterol $=2.31 \pm 0.5 \mathrm{~g} / \mathrm{L}(\mathrm{N}<1.3)$, triglycerides $=1.92$ $\pm 1.26 \mathrm{~g} / \mathrm{L}(\mathrm{N}<1.33)$. For the Wolman patients, laboratory work-up showed an impaired lipid profile, cholesterol $=1.53 \pm 0.87 \mathrm{~g} / \mathrm{L}, \mathrm{HDL}$ cholesterol $=0.15 \pm 0.08 \mathrm{~g} / \mathrm{L}, \mathrm{LDL}$ cholesterol $=1.3 \pm 0.92$ $\mathrm{g} / \mathrm{L}$, triglycerides $=3.26 \pm 1.42 \mathrm{~g} / \mathrm{L}$. 
The main clinical phenotype of Wolman patients was as follows: all patients were from consanguineous parents and presented with hepatomegaly and hypertriglyceridemia, 5/5 showed adrenal calcification, 5/6 presented with anemia, 5/6 with splenomegaly and 5/5 with elevated serum transaminases, 4/6 presented failure to thrive and 4/6 died in infancy, 2/2 vomiting, 2/6 with hypercholesterolemia, 2/5 hepatic fibrosis, 2/5 hepatic steatosis, 1/3 diarrhea, $1 / 3$ abnormality in cardiovascular system and $1 / 5$ with cirrhosis. Regarding CESD patients, 13/13 presented with hepatomegaly, 11/11 showed elevated in serum transaminases, 9/9 presented with hypercholesterolemia, 7/11 with hepatic steatosis, 5/7 with hypertriglyceridemia, 3/9 with hepatic fibrosis and 2/10 with splenomegaly (Supplementary Table S2 and Table S3).

Molecular investigation has been conducted in seventeen patients. It yielded 34 mutated alleles (Figure 4 and Supplementary Table S4). Fourteen unique variants have been identified, 7 have been previously reported [9, 25-30]. The common variant, c.894G>A / p.(Gln298=) [9], has been identified in 12 mutated alleles. Seven variants were novel and included four in CESD form: NM_000235:c.340G>T - p.(Asp114Tyr), c.232_243delins - p.(Pro76Alafs*5), c.538+1G>A 6 p.(?), c.647T>G - p.(Leu216*) and three in Wolman disease: c.429-1G>C - p.(?), c.601G>C - p.(Gly201Arg) and c.538G>A - p.(Gly180Ser).

\section{Discussion}

LALD presentation covers a very wide range of clinical severity spanning from a life-threatening pediatric form to a non-specific, slowly progressive adult form.

The progressive lipid accumulation, primarily in hepatocytes and macrophages, underlies the organomegaly, gastro-intestinal dysfunction, liver disease, and dyslipidemia observed in LALD patients. First-line diagnosis relies on LAL enzyme activity assessment using peripheral leukocytes or DBS. The recent development of rapid and reliable fluorimetric method using DBS [10] enabled the screening of large at-risk populations. Molecular study of LIPA gene is indicated to confirm LALD diagnosis. Considering the ever-growing advances in LD therapies, an early and accurate diagnosis of LALD is critical. Unexplained liver disease and/or lipid abnormalities should prompt to assess LAL enzyme activity. Besides, hypercholesterolemic status may be misleading. Indeed, known hypercholesterolemia disorders are often evoked as first-line diagnosis while this pheonotype might be consistent with LALD [31]. 
The results presented here are issued from the diagnostic work-up of patients presenting with clinical or biological signs consistent with LALD, namely unexplained hepatomegaly or increased transaminase activity ( $\geq 1.5$ times the upper reference limit), or abnormal serum lipid profile with or without splenomegaly or gastrointestinal dysfunction or hepatic microvacuolar steatosis/fibrosis/cirrhosis. Nineteen patients have been diagnosed with LALD with an LAL activity below $0.05 \mathrm{nmol} / \mathrm{punch} / \mathrm{h}$ (LALD patients' overall mean $=0.01$ ). No difference has been observed in LAL activity values between CESD and Wolman patients. Six patients were diagnosed with Wolman disease out of 157 children below 2 years old (3.82\%). Regarding CESD, 13 patients were identified out of 4017 individuals older than 2 years of age $(0.32 \%)$. This very low diagnosis yield prompted us to reevaluate the screening criteria considering the clinical and biological features present in the patients characterized in this study and others [32-37]. In our series, hepatic damages were present in all patients and dyslipidemia was noted in 15/15 patients. Besides, Wolman patients presented with additional features such as gastrointestinal disturbances, anemia, failure to thrive, adrenal calcification and premature death. The screening criteria grid proposed here prioritizes liver damages and dyslipidemia and takes into account the main features reported in the literature and in our series. The suggested criteria are presented in Table 1 with a weighted scoring for each feature. To conduct LALD screening, a total score higher than four is required with either (i) at least one liver/spleen and one dyslipidemia feature or (ii) one feature across three different categories. This stringent strategy may allow a sharper, more targeted, and therefore more efficient screening for LALD.

Molecular studies were conducted in 17 patients and succeeded in confirming the molecular basis of LALD in all tested individuals. Fourteen unique variants have been identified; 7 variants have been previously reported [9, 25-30] while 7 were novel ( 3 missense, 1 nonsense, 2 splicing, 1 frameshift variants). The common variant, c.894G>A - p.(Gln298=), accounted for 12 out of 34 mutated alleles (Figure 4) and was exclusively identified in CESD patients. Importantly, severe truncating variants including nonsense, frameshift and splicing variants were associated with both Wolman disease and CESD phenotypes, but the variants identified in Wolman patients were distinct from those associated with CESD phenotype. 


\section{Conclusions}

LALD is a very rare and underdiagnosed condition. This study allowed to identify a series of patients and better tune the screening criteria for LALD in at-risk populations. Besides, our study expands the molecular spectrum knowledge of lipase acid deficiency. The effect of ERT on survival rate and clinical course urges to define efficient diagnosis strategies.

Funding: This research was funded by Alexion Pharmaceuticals.

Data Availability Statement: All the data that support the findings are presented in the manuscript and the supplementary material.

Acknowledgments: The authors are grateful to all patients and their relatives, staff and clinicians for their contributions.

Conflicts of Interest: S.B. received grants for clinical research from Alexion.

\section{References}

[1] J.S. Parham, J.A. Underberg, Lysosomal Acid Lipase Deficiency, Therapeutic Lipidology (2021) 405.

[2] M.S. Brown, J.L. Goldstein, The SREBP pathway: regulation of cholesterol metabolism by proteolysis of a membrane-bound transcription factor, Cell 89(3) (1997) 331-40.

[3] M. Ishigami, F. Ogasawara, K. Nagao, H. Hashimoto, Y. Kimura, N. Kioka, K. Ueda, Temporary sequestration of cholesterol and phosphatidylcholine within extracellular domains of ABCA1 during nascent HDL generation, Scientific reports 8(1) (2018) 6170.

[4] G. Strebinger, E. Müller, A. Feldman, E. Aigner, Lysosomal acid lipase deficiency - early diagnosis is the key, Hepatic medicine : evidence and research 11 (2019) 79-88.

[5] M.N. Ozmen, N. Aygün, I. Kiliç, L. Kuran, B. Yalçin, A. Besim, Wolman's disease: ultrasonographic and computed tomographic findings, Pediatric radiology 22(7) (1992) 541-2.

[6] G.M. Pastores, D.A. Hughes, Lysosomal Acid Lipase Deficiency: Therapeutic Options, Drug design, development and therapy 14 (2020) 591-601.

[7] P. Lohse, S. Maas, P. Lohse, M. Elleder, J.M. Kirk, G.T. Besley, D. Seidel, Compound heterozygosity for a Wolman mutation is frequent among patients with cholesteryl ester storage disease, Journal of lipid research 41(1) (2000) 23-31.

[8] G. Del Angel, A.T. Hutchinson, N.K. Jain, C.D. Forbes, J. Reynders, Large-scale functional LIPA variant characterization to improve birth prevalence estimates of lysosomal acid lipase deficiency, Human mutation 40(11) (2019) 2007-2020.

[9] S. Muntoni, H. Wiebusch, H. Funke, E. Ros, U. Seedorf, G. Assmann, Homozygosity for a splice junction mutation in exon 8 of the gene encoding lysosomal acid lipase in a Spanish kindred with cholesterol ester storage disease (CESD), Human genetics 95(5) (1995) 491-4.

[10] J. Hamilton, I. Jones, R. Srivastava, P. Galloway, A new method for the measurement of lysosomal acid lipase in dried blood spots using the inhibitor Lalistat 2, Clinica chimica acta; international journal of clinical chemistry 413(15-16) (2012) 1207-10. 
[11] Z. Lukacs, M. Barr, J. Hamilton, Best practice in the measurement and interpretation of lysosomal acid lipase in dried blood spots using the inhibitor Lalistat 2, Clinica chimica acta; international journal of clinical chemistry 471 (2017) 201-205.

[12] B. Sudrie-Arnaud, F. Marguet, S. Patrier, J. Martinovic, F. Louillet, F. Broux, F. Charbonnier, H. Dranguet, S. Coutant, M. Vezain,

R. Lanos, A. Tebani, M. Fuller, F. Lamari, P. Chambon, A.C. Brehin, L. Trestard, I. Tournier, S. Marret, E. Verspyck, A. Laquerriere,

S. Bekri, Metabolic causes of nonimmune hydrops fetalis: A next-generation sequencing panel as a first-line investigation, Clinica chimica acta; international journal of clinical chemistry 481 (2018) 1-8.

[13] E. Muller, B. Brault, A. Holmes, A. Legros, E. Jeannot, M. Campitelli, A. Rousselin, N. Goardon, T. Frébourg, S. Krieger, H. Crouet, A. Nicolas, X. Sastre, D. Vaur, L. Castéra, Genetic profiles of cervical tumors by high-throughput sequencing for personalized medical care, Cancer Med 4(10) (2015) 1484-93.

[14] P.C. Ng, S. Henikoff, Predicting deleterious amino acid substitutions, Genome Res 11(5) (2001) 863-74.

[15] I.A. Adzhubei, S. Schmidt, L. Peshkin, V.E. Ramensky, A. Gerasimova, P. Bork, A.S. Kondrashov, S.R. Sunyaev, A method and server for predicting damaging missense mutations, Nature methods 7(4) (2010) 248-9.

[16] J.M. Schwarz, C. Rödelsperger, M. Schuelke, D. Seelow, MutationTaster evaluates disease-causing potential of sequence alterations, Nature methods 7(8) (2010) 575-6.

[17] K.A. Jagadeesh, A.M. Wenger, M.J. Berger, H. Guturu, P.D. Stenson, D.N. Cooper, J.A. Bernstein, G. Bejerano, M-CAP eliminates a majority of variants of uncertain significance in clinical exomes at high sensitivity, Nature genetics 48(12) (2016) 1581-1586.

[18] F.O. Desmet, D. Hamroun, M. Lalande, G. Collod-Béroud, M. Claustres, C. Béroud, Human Splicing Finder: an online bioinformatics tool to predict splicing signals, Nucleic acids research 37(9) (2009) e67.

[19] G. Yeo, C.B. Burge, Maximum entropy modeling of short sequence motifs with applications to RNA splicing signals, J Comput Biol 11(2-3) (2004) 377-94.

[20] M.G. Reese, F.H. Eeckman, D. Kulp, D. Haussler, Improved splice site detection in Genie, J Comput Biol 4(3) (1997) 311-23.

[21] M. Pertea, X. Lin, S.L. Salzberg, GeneSplicer: a new computational method for splice site prediction, Nucleic acids research 29(5) (2001) 1185-90

[22] R. Leman, P. Gaildrat, G. Le Gac, C. Ka, Y. Fichou, M.P. Audrezet, V. Caux-Moncoutier, S.M. Caputo, N. Boutry-Kryza, M. Léone, S. Mazoyer, F. Bonnet-Dorion, N. Sevenet, M. Guillaud-Bataille, E. Rouleau, B. Bressac-de Paillerets, B. Wappenschmidt, M. Rossing, D. Muller, V. Bourdon, F. Revillon, M.T. Parsons, A. Rousselin, G. Davy, G. Castelain, L. Castéra, J. Sokolowska, F. Coulet, C. Delnatte, C. Férec, A.B. Spurdle, A. Martins, S. Krieger, C. Houdayer, Novel diagnostic tool for prediction of variant spliceogenicity derived from a set of 395 combined in silico/in vitro studies: an international collaborative effort, Nucleic acids research 46(15) (2018) 79137923.

[23] L. Cartegni, J. Wang, Z. Zhu, M.Q. Zhang, A.R. Krainer, ESEfinder: A web resource to identify exonic splicing enhancers, Nucleic acids research 31(13) (2003) 3568-71.

[24] S. Richards, N. Aziz, S. Bale, D. Bick, S. Das, J. Gastier-Foster, W.W. Grody, M. Hegde, E. Lyon, E. Spector, K. Voelkerding, H.L. Rehm, Standards and guidelines for the interpretation of sequence variants: a joint consensus recommendation of the American College of Medical Genetics and Genomics and the Association for Molecular Pathology, Genet Med 17(5) (2015) 405-24.

[25] C. Aslanidis, S. Ries, P. Fehringer, C. Büchler, H. Klima, G. Schmitz, Genetic and biochemical evidence that CESD and Wolman disease are distinguished by residual lysosomal acid lipase activity, Genomics 33(1) (1996) 85-93.

[26] I. Chabchoub, H. Boudabbous, I. Maaloul, R. Ben Abdelaziz, A. Ben Chehida, L. Ayadi, T. Kamoun, N. Tebib, T. Boudaouara, S. Bekri, M. Hachicha, Hemophagocytic Lymphohistiocytosis: A Rare Complication of an Ultrarare Lysosomal Storage Disease, Journal of pediatric hematology/oncology 42(4) (2020) 310-312.

[27] I. Redonnet-Vernhet, M. Chatelut, R. Salvayre, T. Levade, A novel lysosomal acid lipase gene mutation in a patient with cholesteryl ester storage disease, Human mutation 11(4) (1998) 335-6.

[28] F. Tinsa, M. Ben Romdhane, H. Boudabous, I. Bel Hadj, I. Brini, N. Tebib, H. Louati, S. Bekri, K. Boussetta, A Novel Mutation c.153 C>A in a Tunisian Girl With Wolman Disease and Unusual Presentation: Hemophagocytic Lymphohistiocytosis, Journal of pediatric hematology/oncology 41(3) (2019) e193-e196. 
[29] Y. Valles-Ayoub, S. Esfandiarifard, D. No, P. Sinai, Z. Khokher, M. Kohan, T. Kahen, D. Darvish, Wolman disease (LIPA p.G87V) genotype frequency in people of Iranian-Jewish ancestry, Genetic testing and molecular biomarkers 15(6) (2011) 395-8.

[30] O. Zschenker, N. Jung, J. Rethmeier, S. Trautwein, S. Hertel, M. Zeigler, D. Ameis, Characterization of lysosomal acid lipase mutations in the signal peptide and mature polypeptide region causing Wolman disease, Journal of lipid research 42(7) (2001) 103340.

[31] S.W. Fouchier, J.C. Defesche, Lysosomal acid lipase A and the hypercholesterolaemic phenotype, Current opinion in lipidology 24(4) (2013) 332-8.

[32] D.L. Bernstein, H. Hülkova, M.G. Bialer, R.J. Desnick, Cholesteryl ester storage disease: review of the findings in 135 reported patients with an underdiagnosed disease, J Hepatol 58(6) (2013) 1230-43.

[33] B.K. Burton, P.B. Deegan, G.M. Enns, O. Guardamagna, S. Horslen, G.K. Hovingh, S.J. Lobritto, V. Malinova, V.A. McLin, J. Raiman, M. Di Rocco, S. Santra, R. Sharma, J. Sykut-Cegielska, C.B. Whitley, S. Eckert, V. Valayannopoulos, A.G. Quinn, Clinical Features of Lysosomal Acid Lipase Deficiency, Journal of pediatric gastroenterology and nutrition 61(6) (2015) 619-25.

[34] B.K. Burton, N. Silliman, S. Marulkar, Progression of liver disease in children and adults with lysosomal acid lipase deficiency, Curr Med Res Opin 33(7) (2017) 1211-1214.

[35] E.P. Hoffman, M.L. Barr, M.A. Giovanni, M.F. Murray, Lysosomal Acid Lipase Deficiency, in: M.P. Adam, H.H. Ardinger, R.A. Pagon, S.E. Wallace, L.J.H. Bean, G. Mirzaa, A. Amemiya (Eds.), GeneReviews(®), University of Washington, Seattle

[36] Ž. Reiner, O. Guardamagna, D. Nair, H. Soran, K. Hovingh, S. Bertolini, S. Jones, M. Ćorić, S. Calandra, J. Hamilton, T. Eagleton,

E. Ros, Lysosomal acid lipase deficiency--an under-recognized cause of dyslipidaemia and liver dysfunction, Atherosclerosis 235(1) (2014) 21-30.

[37] C. Ruiz-Andrés, E. Sellés, A. Arias, L. Gort, Lysosomal Acid Lipase Deficiency in 23 Spanish Patients: High Frequency of the Novel c.966+2T>G Mutation in Wolman Disease, JIMD Rep 37 (2017) 7-12.

\section{Legends}

Figure 1. Cohort description.

Figure 2. LAL enzymatic activity distribution between Non-LALD, CESD and Wolman patients. The panel to the left is a zoom on low activity values.

Figure 3. Clinical phenotype overview of the CESD and Wolman patients. Blank box means that the status of clinical feature has not been reported. ${ }^{*}$ Hypercholesterolemia is defined with either increased total cholesterol or LDL cholesterol concentrations.

Figure 4. Molecular variations of the seventeen investigated cases describing seven novel and seven previously reported variants. The number of mutated alleles is indicated in the circle. The size of the bubble is proportional to the number of mutated alleles. 
Hepatomegaly Elevated Transmaniases Splenomegaly Hepatic steatosis Cirrhosis Heaptic fibrosis

Death in infancy Failure to thrive Anemia Adrenal calcification Hypercholesterolemia Hypertriglyceridemia Cardiovascular Abnormality
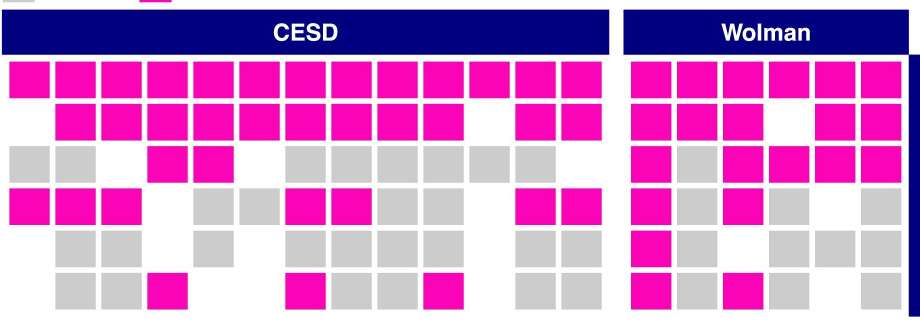

Liver/Spleen
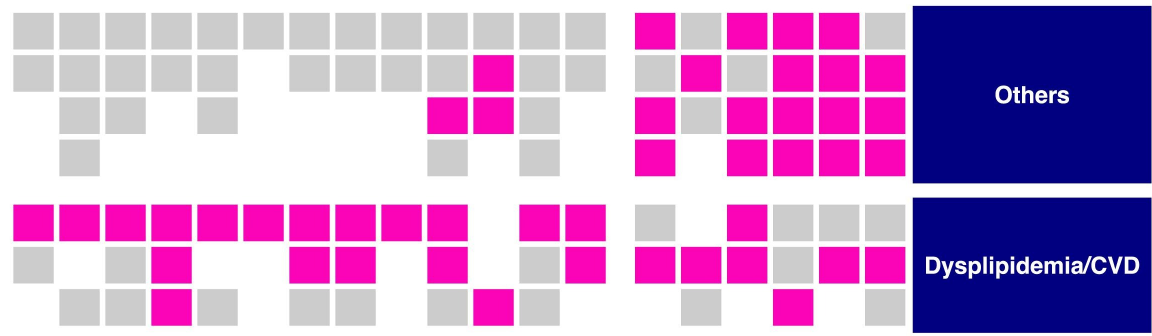

Dysplipidemia/CVD

\section{Diarrhea Vomiting}
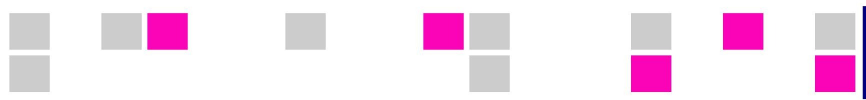

Gastrointestinal

Consanguinous
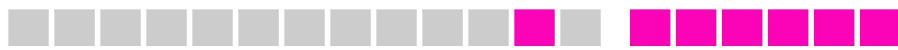

Consanguinity

Enzymotherapy

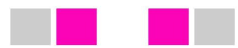
$\begin{array}{lllllllllllll}\text { P2 } & \text { P3 } & \text { P5 } & \text { P6 } & \text { P7 } & \text { P8 } & \text { P9 } & \text { P10 } & \text { P11 } & \text { P13 } & \text { P14 } & \text { P17 } & \text { P18 }\end{array}$

$\begin{array}{llllll}\text { P1 } & \text { P4 } & \text { P12 } & \text { P15 } & \text { P16 } & \text { P19 }\end{array}$ 


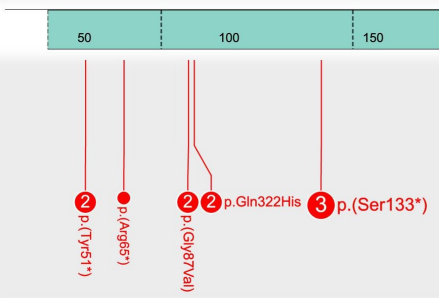

200

250

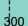

300

2) 12 p.(GIn298=)

Published

7 variants 
Table 1. Screening criteria.

\begin{tabular}{llc}
\hline Categories & Features & Score \\
\hline Liver/Spleen & Hepatomegaly & 3 \\
& Elevated transaminases $(>1.5 \mathrm{~N})$ & 3 \\
& Microvacuolar Steatosis & 3 \\
& Fibroris/Cirrhosis & 2 \\
& Splenomegaly & 1 \\
\hline Dysplipidemia & LDL $>1.3$ g/L (under lipid lowering treatment) & 3 \\
& HDL $<0.45$ g/L & 3 \\
\hline Gastrointestinal & Diarrhea/Vomiting & 3 \\
& Ascitis & 1 \\
\hline Other & Failure to thrive & 3 \\
& Adrenal calcification & 3 \\
& Abdominal distension/pain & 1 \\
& Thrombopenia & 1 \\
\hline & Anemia & 1 \\
\hline
\end{tabular}

404

405 


\section{Highlights}

- A screening of 4174 at-risk LALD patients is reported

- Nineteen patients including thirteen CESD and six Wolman have been identified

- Thirty-three mutated alleles have been identified including fourteen unique variants have been characterized, 7 of which are novel.

- A new LALD screening criteria grid based on the clinical/biological data is proposed. 